\title{
VALORES SINTÁCTICO-DISCURSIVOS DE LAS ETIQUETAS EN TWITTER
}

\author{
Ana Mancera Rueda y Ana Pano Alamán \\ Universidad de Sevilla, Università di Bologna \\ anamancera en us es, ana.pano en unibo it
}

Resumen

En este trabajo sobre el discurso político en la red de microblogging Twitter se presentan los resultados de un análisis cuantitativo y cualitativo de las propiedades sintácticas y discursivas de un corpus de etiquetas o hashtags contenidos en los tuits de diversos políticos españoles, publicados durante la campaña relativa a las elecciones al Parlamento Europeo de 2014. El estudio, basado en los supuestos de la lingüística del discurso, se centra en las relaciones que se establecen entre los rasgos gramaticales de la etiqueta, como su estructura, posición y función sintáctica, y los valores discursivos de la misma, entendidos como posibles indicadores de la intencionalidad del hablante y/o de su presencia en la enunciación, en el marco de la campaña electoral.

Palabras clave: sintaxis, discurso, comunicación política, etiqueta, Twitter

Ana Mancera Rueda y Ana Pano. 2015.

Valores sintáctico-discursivos de las etiquetas en Twitter

Círculo de Lingüística Aplicada a la Comunicación 64, 58-83.

http://www.ucm.es/info/circulo/no64/mancera.pdf

http://revistas.ucm.es/index.php/CLAC

http://dx.doi.org/10.5209/rev_CLAC.2015.v64.51278

(C) 2015 Ana Mancera Rueda y Ana Pano

Círculo de Lingüística Aplicada a la Comunicación (clac)

Universidad Complutense de Madrid. ISSN 1576-4737. http://www.ucm.es/info/circulo 


\begin{abstract}
Syntactic and discursive values of Twitter hashtags

In this paper we focus on political discourse in the microblog Twitter. We present the results of a quantitative-qualitative analysis of the syntactic and discursive properties of a corpus of hashtags in the tweets of different Spanish politicians, published during the campaign for the European Parliament elections in 2014. This study is based on the assumptions of discourse analysis and aims at detecting the relation between the grammatical features of the hashtags, such as structure, position and syntactic functions, and their discursive values within the context of the electoral campaign, the hashtag being an indicator of the speaker's intentionality and modality of enunciation.
\end{abstract}

Keywords: syntax, discourse, political communication, hashtag, Twitter

Índice

1. Introducción, 59

2. Marco teórico, 60

3. Metodología y descripción de datos, 64

4. Análisis cuantitativo en función del número de tuits y de etiquetas, 66

5. Análisis sintáctico y discursivo, 69

6. Conclusiones, 80

Bibliografía, 81

\title{
1. Introducción
}

\#DeLaCalleAEuropa, \#loqueestáenjuegoeselfuturo, \#DebateEuropeasTVE, \#ClaroQuePodemos o \#elpoderdelagente son algunas de las etiquetas utilizadas por distintos políticos españoles durante la campaña electoral que se desarrolló entre el 9 y el 25 de mayo de 2014 con ocasión de las elecciones al Parlamento Europeo. Hoy en día, la etiqueta o hashtag se ha convertido en uno de los dispositivos más utilizados por los usuarios de Twitter, traspasando incluso las fronteras de la red de microblogging gracias a su gran versatilidad. No en vano, la etiqueta permite no solo ahorrar espacio para decir 
más con menos, sino también marcar el tema del tuit como una forma de metadato explícito en el mensaje, que puede aparecer integrado en la estructura sintáctica del enunciado en el que se inserta o bien en los márgenes, al inicio o al final del mensaje, además de tener distintas funciones -informativas, persuasivas, argumentativas, expresivas- en el discurso.

Como se puede inferir de los ejemplos que abren esta introducción, las etiquetas presentan distintos rasgos gramaticales. Pero, además, en función de sus propiedades morfosintácticas, función sintáctica en la estructura en la que se insertan, posición y relación con el resto de elementos lingüísticos del tuit, entre otros aspectos, pueden cumplir distintas funciones en el discurso político en Twitter. A partir de estas consideraciones, nuestro objetivo en este trabajo es profundizar, desde la perspectiva de la lingüística del discurso, en la relación que se establece entre los valores sintácticos de las etiquetas y sus propiedades discursivas en la comunicación política española en la red de microblogging. Así, las preguntas que guían esta investigación son: ¿Qué rasgos sintácticos presentan las etiquetas utilizadas por los políticos españoles durante la campaña electoral al Parlamento europeo? ¿Cuáles son las principales funciones discursivas que tienen las etiquetas en estos mensajes? ¿Es posible establecer una relación entre los rasgos sintácticos de las etiquetas y determinados valores discursivos? Tras revisar algunas cuestiones teóricas relacionadas con estos dispositivos, llevamos a cabo un análisis cuantitativo y cualitativo de las etiquetas presentes en un corpus de tuits elaborado para los fines de este trabajo, que permita responder a las cuestiones planteadas.

\section{Marco teórico}

Gran parte de las investigaciones dedicadas al estudio de las etiquetas en Twitter se basa en los métodos de la lingüística computacional y en análisis estadísticos que buscan identificar de manera automática el contenido de los tuits (Dann 2010), el género al que pertenecen (Shaffer y otros 2013), o la manera cómo se produce la ironía en estos textos breves (Reyes y otros 2012). Otras investigaciones se han ocupado de este dispositivo como indicador del grado de difusión de un determinado tema (Romero y otros 2011; Chew y Eysenbach 2011) y, en otros casos, se han analizado para ver si conllevan innovaciones linguísticas (Cunha y otros 2011) o para averiguar en qué medida 
contribuyen a la difusión de nuevas ideas (Tsur y Rappoport 2012). Si bien estos trabajos aportan datos interesantes sobre los aspectos propiamente lingüísticos de los hashtags, como sus rasgos gramaticales, su posición en el tuit o su valor semántico, estos se consideran solo variables estadísticas para investigar otras cuestiones.

En el ámbito del análisis lingüístico del discurso podemos asumir algunos de los planteamientos de Zappavigna $(2011,2012)$, para quien la etiqueta tiene esencialmente dos funciones: en primer lugar, señala que el hashtag, en la estructura del enunciado, puede indicar una clase, un proceso o un objeto, categorías propias de la gramática sistémico-funcional; en segundo lugar, tiene una función de metadato explícito que se integra en el mensaje para definir el tema del mismo, lo que coloca a Twitter en el mundo del etiquetado colaborativo o folksonomía, es decir, un método de clasificación colaborativa de datos a partir de palabras clave elegidas por los internautas. En este caso, la etiqueta amplía el significado potencial del tuit puesto que, convirtiéndose en un tag que es posible enlazar y buscar en la Red, se relaciona con otros muchos mensajes y discursos, promoviendo lo que la autora denomina ambiente de afiliación (Zappavigna 2011). Gracias al buscador de Twitter, la etiqueta permite recuperar también un tuit o un conjunto de tuits relacionados con el tema que encierra el dispositivo, y seguir la "conversación" que se desarrolla sobre ese mismo tema. Zappavigna define este tipo de contenido como searchable talk o "conversación que puede buscarse", y que fomenta la generación de distintas opiniones alrededor de un mismo tópico. En relación con este aspecto y en el ámbito del discurso político, Davis sostiene lo siguiente: "This renders users' speech as more findable and relatable, which in effect strengthens interpersonal connections and creates a sense of commonality through shared values and interests" (Davis 2013: 18).

A conclusiones similares han llegado estudios recientes sobre el uso de etiquetas en los discursos mediáticos ${ }^{1}$ y políticos en Twitter. En concreto, resultan útiles para nuestros propósitos los planteamientos recogidos en el análisis llevado a cabo por Mancera y Helfrich (2014) sobre el valor propagandístico de la etiqueta en los tuits de empresas,

\footnotetext{
${ }^{1}$ Cfr. Parmelee y Bichard (2012) y Page (2012). Por ejemplo, basándose en los planteamientos de la teoría de la agenda-setting y del framing, Pérez Fumero (2013) lleva a cabo un análisis de contenido de tuits relacionados con la etiqueta \#ElMundoEstaConChavez, en el que demuestra que esta etiqueta, insertada como enunciado independiente en miles de tuits, contribuyó a la construcción de dos tipos de encuadres, uno positivo y otro negativo, sobre la enfermedad de Chávez.
} 
medios, políticos y ciudadanos, relativos a la crisis económica. El estudio muestra que la etiqueta juega un papel fundamental en la conformación ideológica del concepto de crisis. Y es que, a través de hashtags como \#paro o \#desahucios, que poseen connotaciones negativas, los hablantes manifiestan su perspectiva enunciativa. Desde un punto de vista pragmático, etiquetas como \#Eurogrupo o \#banca adquieren un carácter peyorativo cuando aluden a entidades supuestamente responsables de la crisis, mientras que \#stopdesahucios, \#15M o \#escrache actúan como eslóganes que simbolizan la lucha contra las causas y efectos de la misma, pudiendo incitar a la acción (Mancera y Helfrich 2014: 65). En relación con el discurso político, esencialmente propagandístico, las autoras observan que las etiquetas permiten clasificar los tuits en mensajes de apoyo hacia las políticas llevadas a cabo por el gobierno para hacer frente a la crisis, y mensajes de crítica al gobierno por parte de los partidos en la oposición. Asimismo, y esto se observa también en los tuits publicados por empresas y medios de comunicación, mediante las etiquetas los políticos elaboran una retórica de la persuasión para mostrar que están "en primera línea del debate público" (Mancera y Helfrich 2014: 83), incrementar el interés de los ciudadanos por sus mensajes y conseguir su adhesión.

En cuanto a los aspectos formales y gramaticales de las etiquetas analizadas, Mancera y Helfrich apuntan que, a menudo, estas se integran en el mensaje como un sintagma nominal en el que sus lexías pueden presentarse fusionadas, aunque

en los casos en los que la etiqueta aparece al final del mensaje, es decir, sin integración sintáctica alguna, permite que esta sea visualizada de manera más rápida, y al mismo tiempo resume a modo de eslogan el contenido del tuit. (Mancera y Helfrich 2014: 80)

El valor de las etiquetas como eslóganes ${ }^{2}$ y consignas ha sido señalado también en Mancera y Pano (2013), donde se comprueba que la mayor parte de los políticos emplean estrategias de persuasión y seducción similares a las que se observan en otros géneros, como el debate cara a cara y el mitin electoral. Las etiquetas se apoyan a menudo en construcciones semánticamente ambiguas y en términos ambivalentes o poco precisos; vehiculan el apoyo a los miembros del propio partido y la crítica al adversario; e "incitan a hacer", de cara a lograr una reacción en el destinatario, que lo

\footnotetext{
${ }^{2}$ Muchas de las etiquetas utilizadas por los equipos de campaña en las redes sociales presentan algunos de los rasgos atribuidos al eslogan, como brevedad, concisión, simplicidad sintáctica o densidad semántica (cfr. López Eire y de Santiago Guervós 2000).
} 
lleve a identificarse o a respaldar lo planteado por el emisor. Pueden verse, por tanto, como consignas que sirven para reforzar la adhesión a un determinado político o partido, o para criticar su propuesta, generando dinámicas de interacción de muchos a muchos que, según lo asertado, pueden tener consecuencias positivas o negativas para el político. No obstante, las etiquetas no solo se convierten en mecanismos para contribuir a la difusión del mensaje a modo de eslogan perdurable, sino que también se insertan en el enunciado con funciones sintácticas específicas y como marcas de la enunciación.

Por tanto, es posible afirmar con Menna (2012) que los hashtags en Twitter son elementos semióticos complejos, de características formales y funcionales innovadoras que operan en dos niveles: el pragmático-discursivo y el gramatical. Desde un punto de vista discursivo, la autora señala la importancia de la polifonía textual, en la medida en que las etiquetas permiten reproducir y al mismo tiempo "escuchar" distintas voces en relación con un mismo tema. Además, la posibilidad de incluir, a través de los hashtags, un coro de voces que sostienen o refutan lo que decimos, se convierte en una estrategia al servicio de la argumentación y de la expresión de la opinión (Menna 2012: 34).

El análisis llevado a cabo por esta investigadora sobre el uso de etiquetas relacionadas con el \#12M15M y utilizadas por los movimientos de protesta contra la crisis económica muestra que estas tenían, por un lado, funciones organizativas dentro de Twitter, para clasificar los contenidos en temas y subtemas; y, fuera de la red social, servían para indicar, entre otras cosas, los lugares de las manifestaciones organizadas por el movimiento $15 \mathrm{M}$; por otro lado, desempeñaron funciones comunicativas que bien pueden asociarse a las de los lemas, reclamos, llamamientos a la acción y denuncias.

En lo que concierne a los valores gramaticales de las mismas, Menna (2012: 49) destaca que en su corpus estas presentaban una gran heterogeneidad. Así, algunas poseían una estructura oracional y solían incluirse en el tuit como proposiciones subordinadas, mientras que otras constituían sintagmas más o menos extensos, que se insertaban en la oración con diversas funciones sintácticas, de modo que era posible identificar distintas dimensiones en las relaciones que se establecían entre el dispositivo y el resto de los elementos lingüísticos del mensaje. De hecho, como señala esta autora, el signo \# es un "elemento semiótico relevante" que establece relaciones sintagmáticas con el resto de los constituyentes del tuit. Así, pueden identificarse tres tipos de relaciones: la 
sintagmática interna, que se refiere a la disposición de las formas escritas vinculadas entre sí y con otras formas dentro de la misma secuencia lingüística; la sintagmática externa, que hace referencia a las relaciones posibles entre formas escritas y el espacio exterior en el cual está situado el espacio gráfico (por ejemplo, cuando la etiqueta se transforma en hipervínculo para acceder a todos los tuits que la contienen); y la sintagmática contextual, que es la relación que se establece entre una unidad significante, en este caso un tuit, y el contexto situacional al cual remite, generando nuevos significados que apelan a los conocimientos compartidos por el hablante y el oyente de acuerdo con las condiciones de producción y recepción de los mensajes.

\section{Metodología y descripción de datos}

Para analizar el uso que hicieron de las etiquetas los políticos españoles durante los comicios al Parlamento Europeo de 2014, hemos recopilado todos los mensajes publicados entre el 9 y el 25 de mayo de dicho año (es decir, desde el comienzo de la campaña electoral hasta el día en el que tuvieron lugar las votaciones en España) en treinta perfiles de Twitter. Entre estos hemos seleccionado en primer lugar los de los cinco partidos más votados ${ }^{3}$ : el Partido Popular (@PPopular), el Partido Socialista Obrero Español (@PSOE), La Izquierda Plural ${ }^{4}$ (@iunida), Podemos (@ahorapodemos) y Unión Progreso y Democracia (@UPyD).

Por otra parte, conforman también nuestro corpus los mensajes publicados en tales fechas por los principales candidatos presentados por estas formaciones. Así, hemos estudiado el perfil del eurodiputado del Partido Popular Miguel Arias Cañete (@Canete 2014_); el de Elena Valenciano Martínez-Orozco (@ElenaValenciano), eurodiputada del Partido Socialista; el de Willy Enrique Meyer Pleite (@willymeyerIU), de La

\footnotetext{
${ }^{3}$ Cfr. por ejemplo la página web "Elecciones Europeas. 25 mayo 2014”, elaborada por el Ministerio del Interior del Gobierno de España: <http://elecciones.mir.es/resultados2014/99PE/DPE99999TO.htm>. Última consulta: 13-08-2014.

${ }^{4}$ Esta es una coalición constituida para concurrir a dichos comicios de 2014 por diversos partidos, como Izquierda Unida (IU), Confederación de Los Verdes y Construyendo la Izquierda-Alternativa Socialista, entre otras formaciones de ámbito autonómico. Más información puede obtenerse en su página web, disponible en: <http://oncediputados.blogspot.com.es/2012/11/la-izquierda-plural-pagina-oficial.html>. Última consulta: 13-08-2014. En Twitter hemos podido localizar dos perfiles asociados al nombre de dicha coalición: Izquierda Plural (@izquierdapl) e izquierda plural (@izquierda_pl). Sin embargo, ninguno de ellos parece ser oficial, pues no contiene mensajes publicados en las fechas en las que tuvo lugar la campaña electoral. Además, el uso de la herramienta de búsqueda de la propia red de microblogging remite a la cuenta del principal partido de los que componen la coalición: IUzquierda Unida (@iunida).
} 
Izquierda Plural; el de Pablo Iglesias Turrión (@Pablo_Iglesias_), de Podemos; y el del candidado que concurría en el tercer lugar ${ }^{5}$ de la lista electoral de Unión Pogreso y Democracia, Fernando Maura Barandiarán (@FMauraB).

Por último, con el objeto de averiguar en qué medida otros representantes de dichos partidos hicieron o no uso de los mismos hashtags que los líderes más directamente involucrados en los comicios europeos, se han analizado además las cuentas de veinte de los políticos más activos en esta red social. Al igual que en Pano y Mancera (2014), nos hemos servido también aquí para la selección de nuestro corpus de los estudios sobre la actividad en Twitter de los parlamentarios españoles de la X Legislatura llevados a cabo por el experto en comunicación política y redes sociales David Álvarez ${ }^{6}$. Si bien en esta ocasión hemos optado por dejar de lado los perfiles de los miembros del Senado, para analizar únicamente los de dieciséis congresistas (cuatro por cada una de las fuerzas políticas con representación parlamentaria mencionadas anteriormente): Teodoro García Egea (@TeoGarciaEgea), Conrado Escobar Las Heras (@conrado_escobar), María del Carmen Rodríguez Maniega (@carmenmaniega) y Esteban González Pons (@gonzalezpons), del PP; José Martínez Olmos (@PmOlmos), Patricia Hernández Gutiérrez (@PatriciaHdezGut), Gracia Fernández Moya (@gracitacary) y José Ignacio Sánchez Amor (@NachoSAmor), del PSOE; Alberto Garzón Espinosa (@agarzon), Gaspar Llamazares Trigo (@Gllamazares), Cayo Lara Moya (@ cayo_lara) y Chesús Yuste Cabello (@ChesusYuste), de La Izquierda Plural; y Carlos Martínez Gorriarán (@cmgorriaran), Álvaro Anchuelo Crego (@aanchuelo), Irene Lozano Domingo (@lozanoirene) y Toni Cantó García (@Tonicanto1), de $\mathrm{UPyD}^{7}$. Asimismo, con el fin de consignar el mismo número de políticos de cada uno de los cinco partidos seleccionados, y al no contar Podemos con representación parlamentaria, se han recopilado también los mensajes publicados por otros cuatro eurodiputados de esta formación ${ }^{8}$ : Teresa Rodríguez-Rubio Vázquez (@TeresaRodr),

\footnotetext{
${ }^{5}$ Ni Francisco Sosa Wagner ni Maite Pagazaurtundua Ruiz cuentan con perfil en esta red social.

${ }^{6}$ Una selección de sus interesantes trabajos puede extraerse de su bitácora "Parlamento 2.0". Disponible en: <http://parlamento20.es/david-alvarez>. Última consulta: 13-08-2014. No obstante, los resultados de su análisis de la actividad de cada parlamentario en Twitter, teniendo en cuenta tanto el número de tuits enviados como la actividad regular de su perfil, no han sido publicados aún, sino que nos los ha facilitado mediante una comunicación privada el propio investigador, a quien queremos dar las gracias por toda la ayuda prestada.

${ }^{7}$ La portavoz de dicho partido, la diputada Rosa Díez González, no cuenta con perfil en Twitter, aunque sí lo tiene en otra red social, como es Facebook: <https://www.facebook.com/Rosa.Diez.Gonzalez>. Última consulta: 13-08-2014.

${ }^{8}$ Cinco fueron los escaños obtenidos por esta fuerza política en las elecciones al Parlamento de 2014.
} 
Lola Sánchez (@LolaPodemos), Pablo Echenique-Robba (@pnique) y Tania González Peñas (@TaniaGonzalezPs).

El uso de la herramienta de analítica social Topsy Pro Analytics nos ha permitido recuperar todos los mensajes publicados en estos treinta perfiles entre el 9 y el 25 de mayo de 2014, que ascienden a un total de 4.483 tuits. Sin embargo, para nuestro estudio de las etiquetas escogidas por los políticos españoles poca relevancia tienen aquellos textos fruto del mero retuit o reenvío de mensajes redactados por otros usuarios. De ahí que hayamos prescindido en nuestra investigación de un pequeño número tuits (76 mensajes) similares al siguiente:

Toni Cantó (@Tonicanto1).“:-) RT @ fernandollopis Con@ @onicanto1 Rumbo a la gala inaugural del Festival de Cine de \#Alicante”. 24-05-2014. Tuit.

Por tanto, nuestro corpus analítico se compone de 4.407 tuits, en los cuales se han empleado un total de 1.970 etiquetas. Hay que tener en cuenta que no existe una correspondencia exacta entre el número de tuits y el de etiquetas, ya que no todos los mensajes contienen hashtags (1.582 cf. tabla 1). Además, es frecuente que en un mismo texto aparezcan varias etiquetas,

Willy Meyer (@willymeyerIU). "El cambio empieza \#25M La gente no puede quedarse en casa. Ahora no. El \#25M debe ser la gran batalla de las batallas \#europeas2014 @iucyl”. 14-05-2014. Tuit.

que a veces contraen relaciones sintagmáticas entre sí y con el resto de los constituyentes oracionales del mensaje:

Pablo Iglesias (@Pablo_Iglesias). "Sin el enorme gasto en publicidad de los grandes partidos y \#SinBancosPodemos dar la gran sorpresa el \#25M. Colabora: http://crowd funding.podemos.info/". 19-05-2014. Tuit.

Por último, no hemos tenido en cuenta si cada uno de los hashtags utilizados llegó a convertirse en trending topic, ya que dicho aspecto resulta escasamente relevante para nuestra investigación.

\section{Análisis cuantitativo en función del número de tuits y de etiquetas}

Antes de abordar el análisis discursivo del tipo de hashtags que fueron utilizados durante los comicios al Parlamento Europeo de 2014, hemos tratado de responder a los siguientes interrogantes: ¿Cómo fue la actividad en Twitter de cada uno de los cinco partidos seleccionados? ¿En qué medida hicieron uso de dicha red social los representantes de estas fuerzas políticas? ¿Qué representatividad tienen las etiquetas en 
los mensajes de nuestro corpus? La siguiente tabla resume los resultados de esta primera aproximación de carácter cuantitativo.

\begin{tabular}{|c|c|c|c|c|c|c|}
\hline usuarios & 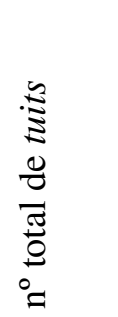 & $\begin{array}{l}\stackrel{\Xi}{\Xi} \\
: \\
:\end{array}$ & 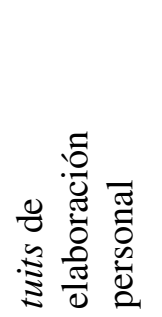 &  & 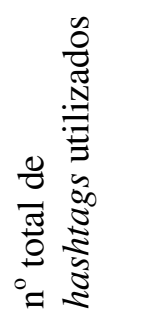 &  \\
\hline PP & 360 & 0 & 360 & 280 & 402 & $111,66 \%$ \\
\hline M. Arias Cañete & 31 & 0 & 31 & 11 & 12 & $38,70 \%$ \\
\hline T. García & 55 & 5 & 50 & 13 & 19 & $38 \%$ \\
\hline C. Escobar & 134 & 0 & 134 & 1 & 1 & $0,74 \%$ \\
\hline C. Rodríguez & 36 & 0 & 36 & 17 & 23 & $63,88 \%$ \\
\hline E. González Pons & 90 & 0 & 90 & 40 & 41 & $45,55 \%$ \\
\hline PSOE & 307 & 4 & 303 & 154 & 56 & $18,48 \%$ \\
\hline E. Valenciano & 159 & 0 & 159 & 88 & 107 & $67,29 \%$ \\
\hline J. Martínez & 96 & 0 & 96 & 12 & 13 & $13,54 \%$ \\
\hline P. Hernández & 299 & 0 & 299 & 17 & 25 & $8,36 \%$ \\
\hline G. Fernández & 24 & 2 & 22 & 6 & 6 & $27,27 \%$ \\
\hline J. I. Sánchez & 73 & 0 & 73 & 4 & 4 & $5,47 \%$ \\
\hline IU & 377 & 12 & 365 & 251 & 318 & $87,12 \%$ \\
\hline W. Meyer & 172 & 0 & 172 & 98 & 137 & $79,65 \%$ \\
\hline A. Garzón & 136 & 0 & 136 & 8 & 9 & $6,61 \%$ \\
\hline G. Llamazares & 239 & 14 & 225 & 9 & 9 & $4 \%$ \\
\hline C. Lara & 94 & 0 & 94 & 30 & 31 & $32,97 \%$ \\
\hline C. Yuste & 77 & 0 & 77 & 70 & 90 & $116,88 \%$ \\
\hline Podemos & 387 & 3 & 384 & 154 & 178 & $46,35 \%$ \\
\hline P. Iglesias & 178 & 9 & 169 & 56 & 63 & $37,27 \%$ \\
\hline T. Rodríguez & 63 & 0 & 63 & 15 & 17 & $26,98 \%$ \\
\hline L. Sánchez & 13 & 0 & 13 & 6 & 6 & $46,15 \%$ \\
\hline P. Echenique & 101 & 0 & 101 & 25 & 25 & $24,75 \%$ \\
\hline T. González & 24 & 0 & 24 & 10 & 10 & $41,66 \%$ \\
\hline UPyD & 313 & 9 & 304 & 151 & 304 & $100 \%$ \\
\hline F. Maura & 20 & 1 & 19 & 9 & 11 & $57,89 \%$ \\
\hline C. Martínez & 390 & 3 & 387 & 18 & 18 & $4,65 \%$ \\
\hline A. Cantó & 126 & 9 & 117 & 6 & 6 & $5,12 \%$ \\
\hline I. Lozano & 94 & 5 & 89 & 23 & 29 & $32,58 \%$ \\
\hline A. Anchuelo & 15 & 0 & 15 & 0 & 0 & $0 \%$ \\
\hline TOTAL & 4.483 & 76 & 4.407 & 1.582 & 1.970 & $1.189,57 \%$ \\
\hline
\end{tabular}

Tabla 1: Cómputo de tuits y hashtags 
Como puede apreciarse en la tabla 1, en general fueron los perfiles oficiales de los partidos políticos los que más mensajes publicaron. Algo que no debe extrañarnos si tenemos en cuenta que lo habitual es que estos sean gestionados por un grupo de expertos en redes sociales, conocidos también como community manager ${ }^{9}$. Ignoramos si en las elecciones al Parlamento Europeo de 2014 la gestión de las campañas de los distintos partidos fue similar, pero las cifras que presentamos en la Tabla 1 demuestran que las formaciones políticas fueron las que más tuitearon exceptuando un caso, el del parlamentario de UPyD Carlos Martínez Gorriarán, que es el que mayor número de mensajes publicó a lo largo de los diecisiete días contabilizados. En concreto, difundió un total de 387 tuits fruto de su elaboración personal. Muy similar es la cifra de los mensajes publicados por Podemos (384) y, a continuación, a mayor distancia, le siguen IU (365), PP (360), UPyD (304) y PSOE (303). Precisamente, al uso activo ${ }^{10}$ de las redes sociales atribuyeron los medios de comunicación una buena parte del triunfo electoral de Podemos, un partido de reciente creación ${ }^{11}$. Precisamente los cinco representantes de este partido fueron los que más mensajes enviaron (un total de 704), seguidos por los de PSOE (649) y UPyD (627). A mayor distancia se sitúan los de Podemos $^{12}$ y PP, pues publicaron casi la mitad de tuits (370 y 341, respectivamente).

Los perfiles oficiales de algunos de los partidos políticos fueron también los que más utilizaron las etiquetas en cada uno de sus mensajes. Nos estamos refiriendo a los de PP (111,66\%), UPyD (100\%) e IU (87,12\%). No es este el caso de Podemos (46,35\%) ni del PSOE (18,48\%), en los que la presencia porcentual de hashtags es mucho más reducida. Entre los políticos, fueron los representantes de IU Chesús Yuste (116,88\%) y

\footnotetext{
${ }^{9}$ Además, ya en Mancera y Pano (2013) explicamos cómo en el caso de los candidatos de los partidos mayoritarios que concurrieron a las elecciones generales de 2011 fueron también sus equipos los autores de la mayor parte de los tuits publicados en sus cuentas personales.

${ }^{10}$ Según explicaba en una entrevista Rita Maestre (una de las quince community manager con las que cuenta esta formación): "Desde el inicio sabíamos que tanto Twitter como Facebook eran la clave". Sin embargo, a juzgar por los datos de nuestro corpus, esta preocupación por las redes sociales no parecía ser compartida por los cabeza de lista de los partidos mayoritarios, pues el perfil de Elena Valenciano se sitúa en el lugar once en nuestra lista de aquellos con mayor actividad en Twitter (159 mensajes) y muy por detrás, en el puesto vigésimo quinto, se encuentra el de Miguel Arias Cañete, quien únicamente publicó 31 mensajes a lo largo de toda la campaña electoral. Solo Willy Meyer y Pablo Iglesias, los candidatos de IU y Podemos, se hallan entre los diez perfiles más activos de nuestro corpus, si bien estos tuitearon la mitad de mensajes que sus respectivos partidos (172 y 169). Además, por delante de ellos se sitúan la diputada socialista Patricia Hernández (299) y Gaspar Llamazares, de IU (225).

${ }^{11}$ Fue inscrito en el registro de partidos el 11 de marzo de 2014, poco más de dos meses antes de las elecciones.

${ }^{12}$ La gran actividad desarrollada por Pablo Iglesias en Twitter contrasta mucho con la escasa presencia de otros candidatos de su mismo partido, como Tania González y Lola Sánchez, que solo publicaron 24 y 13 mensajes respectivamente.
} 
Willy Meyer $(79,65 \%)$ los que más etiquetas utilizaron porcentualmente. A continuación les sigue Elena Valenciano (67,29\%). Curiosamente, el diputado de UPyD Carlos Martínez Gorriarán, que ya hemos demostrado que es quien mayor número de tuits publica, apenas incluye hashtags en sus mensajes (4,65\%).

Por último, hemos calculado también la media de uso de las etiquetas en los mensajes de los representantes políticos, excluyendo de dicho cómputo las cuentas oficiales de los partidos, que se sitúa en el 38,84\%. Por encima de dicha cifra se encuentran los perfiles de los líderes de IU (48,02\%). A continuación les siguen los de PP $(37,38 \%)$ y Podemos $(35,36 \%)$. Muy por debajo de la media se hallan los de PSOE $(24,39 \%)$ y UPyD $(20,05 \%)$. Tras esta primera aproximación cuantitativa a nuestro corpus, nos centraremos a continuación en el análisis cualitativo de los hashtags empleados.

\section{Análisis sintáctico y discursivo}

En nuestro corpus hemos localizado un total de 253 etiquetas diferentes. No todas guardan una relación directa con los comicios europeos, como \#Quino, el hashtag con el que algunos políticos mostraron su admiración por el creador de Mafalda, a raíz de la noticia de que se le había concedido el Premio Príncipe de Asturias de Comunicación y Humanidades,

Elena Valenciano (@ElenaValenciano). "Bravo por \#Quino Premio Principe de Asturias! Mafalda salta de contenta y yo con ella". 21-05-2014. Tuit.

\#Diadeinternet, el utilizado para recordar que el 17 de mayo se celebraba el Día mundial de Internet,

Esteban González Pons (@gonzalezpons). “\#DiadeInternet contra la violencia también en Internet. Redes para unir no para romper”. 17-05-2014. Tuit.

o \#AupaAtleti, con el que algunos diputados pretendían infundir ánimos a su equipo:

Teodoro García Egea (@TeoGarciaEgea). “Afición del atlético cantando el himno, y nuestro equipo pierde! \#Seny \#AupaAtleti”. 24-05-2014. Tuit.

Vamos a dejar de lado este tipo de etiquetas para centrarnos en el análisis de aquellas que sí tienen vinculación con el período electoral que estamos estudiando, y que fueron las más utilizadas, como puede apreciarse en la tabla 2, en la que recogemos los diez hashtags empleados con mayor frecuencia por los políticos de cada partido: 


\begin{tabular}{|c|c|c|c|c|}
\hline $\mathbf{P P}$ & PSOE & IU & PODEMOS & UPyD \\
\hline \#VotaPP (195) & $\begin{array}{l}\text { \#TuMuevesEurop } \\
\text { a (60) }\end{array}$ & $\begin{array}{l}\text { \#elpoderdelagente } \\
(224)\end{array}$ & $\begin{array}{l}\text { \#YoVotoPodemos } \\
\text { (74) }\end{array}$ & \#euroUPyD (137) \\
\hline \#VotaCañete (122) & $\begin{array}{l}\text { \#CaraACaraTVE } \\
(31)\end{array}$ & $\begin{array}{l}\text { \#PrimaveraEurope } \\
\text { a (53) }\end{array}$ & $\begin{array}{l}\text { \#VotandoPodemos } \\
(53)\end{array}$ & \#SosaWagner (51) \\
\hline $\begin{array}{l}\text { \#lavidaencampaña } \\
\text { (37) }\end{array}$ & $\begin{array}{l}\text { \#AndalucíaMueve } \\
\text { Europa (12) }\end{array}$ & $\begin{array}{l}\text { \#Europeas2014 } \\
(48)\end{array}$ & $\begin{array}{l}\text { \#ClaroQuePodem } \\
\text { os (21) }\end{array}$ & \#MaitePagaza (32) \\
\hline $\begin{array}{l}\text { \#DebateEuropeas } \\
\text { TVE (28) }\end{array}$ & $\begin{array}{l}\text { \#RosasParaEuropa } \\
\text { (12) }\end{array}$ & \#SomosIU (32) & $\begin{array}{l}\text { \#CartaPodemos } \\
\text { (17) }\end{array}$ & $\begin{array}{l}\text { \#DebatEuropeesT } \\
\text { V3 (18) }\end{array}$ \\
\hline $\begin{array}{l}\text { \#CaraACaraTVE } \\
\text { (16) }\end{array}$ & $\begin{array}{l}\text { \#canviemEuropa } \\
(10)\end{array}$ & $\begin{array}{l}\text { \#DebateEuropeas } \\
\text { TVE (28) }\end{array}$ & $\begin{array}{l}\text { \#SinBancosPodem } \\
\text { os (16) }\end{array}$ & $\# 25 \mathrm{M}(10)$ \\
\hline \#GanaCañete (15) & $\begin{array}{l}\text { \#NoAlMachismo } \\
(8)\end{array}$ & \#votaIU (23) & $\begin{array}{l}\text { \#TereAEuropa } \\
(16)\end{array}$ & \#EP2014 (10) \\
\hline $\begin{array}{l}\text { \#PPCVconRajoy } \\
\text { (14) }\end{array}$ & \#VotaPSOE (6) & \#ganalagente (17) & $\begin{array}{l}\text { \#DifundePodemos } \\
\text { (12) }\end{array}$ & $\begin{array}{l}\text { \#CaraACaraTVE } \\
\text { (9) }\end{array}$ \\
\hline $\begin{array}{l}\text { \#CañeteenCOPE } \\
\text { (7) }\end{array}$ & \#knockthevote (4) & \#puroteatro (5) & $\begin{array}{l}\text { \#QueTeneisQueEs } \\
\text { conder (12) }\end{array}$ & $\begin{array}{l}\text { \#PPSOEsonlomis } \\
\text { mo (9) }\end{array}$ \\
\hline $\begin{array}{l}\text { \#loqueestaenjuego } \\
\text { eselfuturo ( } 7)\end{array}$ & \#almeria (3) & $\begin{array}{l}\text { \#europadelasperso } \\
\text { nas (14) }\end{array}$ & $\# 25 \mathrm{M}(8)$ & \#Europa (6) \\
\hline \#Cmin (6) & \#CalleACalle (3) & $\begin{array}{l}\# 17 \text { medidasIU } \\
(13)\end{array}$ & \#Podemos (8) & \#Congreso (5) \\
\hline
\end{tabular}

Tabla 2: etiquetas más utilizadas ${ }^{13}$

Algunos de estos hashtags tienen una finalidad informativa. Por ejemplo, es frecuente que los topónimos se escriban precedidos por la almohadilla para indicar el lugar en el que se celebrará el próximo mitin de un determinado partido:

Pablo Iglesias (@Pablo_Iglesias). “A las 8 comenzamos el acto en \#Sevilla. Vente y díselo a tu gente ;) \#VotandoPodemos pic.twitter.com/SOsZ3egQVj”. 22-05-2014. Tuit.

Si bien en otros casos podría atribuírseles más bien una finalidad que podríamos calificar como contextualizadora, pues los políticos utilizan estos topónimos como etiqueta para especificar el lugar en el que han sido formuladas ciertas declaraciones:

Gracia Fernández (@gracitacary). “Trinidad Jiménez: “Es un lujo una grandísima oportunidad que Almería pueda tener su propia europarlamentaria"\#almeria http://www. noticiasdealmeria.com/noticia/92056/EUROPEAS-2014/Trinidad-Jimenez:-Es-un-lujo-yuna-grandisima-oportunidad-que-Almeria-pueda-tener-su-propia-europarlamentaria.html ...". 23-05-2014. Tuit.

Esa misma función contextualizadora cumple en los siguientes mensajes la etiqueta \#CaraACaraTVE, utilizada por partidos y políticos de distinta tendencia para especificar que las declaraciones reproducidas en un determinado tuit fueron formuladas durante el

\footnotetext{
${ }^{13}$ Las cifras que incluimos a continuación de cada etiqueta son el resultado de la suma del número de veces que esta aparece en el perfil oficial de cada partido y en los de los diputados y candidatos de esta fuerza política durante el periodo de tiempo analizado.
} 
debate entre Miguel Arias Cañete y Elena Valenciano retransmitido por Televisión Española:

Carmen R. Maniega (@carmenmaniega). “Cañete a Valenciano: \#CaraACaraTVE. Le moleste o no, este gobierno ha creado empleo". 15-05-2014. Tuit.

Partido Popular (@PPopular). “@@Canete2014_ "La mejor política social es la que se basa en el crecimiento económico y el empleo" \#GanaCañete \#CaraACaraTVE”. 15-052014. Tuit.

Incluso durante el transcurso del debate, desde el perfil de ambos candidatos sus equipos enviaban mensajes que incluían dicha etiqueta para destacar los aspectos más relevantes de su discurso, de forma que sus puntos de vista pudieran llegar también a aquellos seguidores que no estaban contemplando el debate por televisión,

Elena Valenciano (@ElenaValenciano). "Sólo hay una persona con derecho a decidir sobre la maternidad de una mujer: ella misma. \#CaraACaraTVE pic.twitter.com/ M9nt4OLqhe". 15-05-2014. Tuit.

o para complementar sus aserciones con gráficos que sustentaran sus argumentos:

Miguel Arias Cañete (@MAC_europa). “Elena Valenciano vuelve a olvidar un dato: la evolución de la pobreza infantil durante su gobierno \#CaraACaraTVE pic.twitter. com/HIcRunj3mU”. 15-05-2014. Tuit.

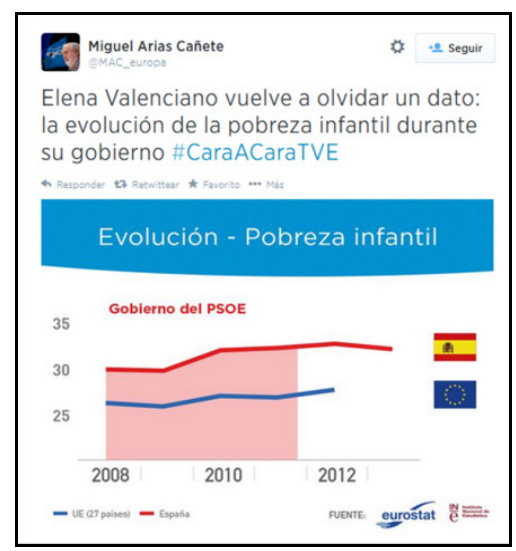

Por otra parte, hemos observado que desde la cuenta oficial de UPyD en ocasiones se reproducía en forma de etiqueta el sujeto de la enunciación de un fragmento de discurso referido:

UPyD (@UPyD). “Tenemos que intentar que las personas que vienen a España no vivan en Ghetos, tampoco seamos angelicales' \#MaitePagaza @UPyD @EspejoPublico". 2305-2014. Tuit. 
Podríamos decir que en este caso el hashtag actúa como señal demarcativa de una cita ${ }^{14}$. Si bien hemos observado que esta función no resulta muy frecuente en nuestro corpus, pues lo habitual para identificar al sujeto enunciador de un fragmento de discurso reproducido es o bien mencionar su nombre propio (sin utilizar ningún tipo de etiqueta),

PSOE (@PSOE). “G.Zarrías:Cañete dijo una verdad, ‘yo no soy un melón sin catar'. Andalucía perdió una buena parte de recursos en la negociación de la PAC". 19-05-2014. Tuit.

o bien el de su perfil de Twitter:

Izquierda Unida (@iunida).""Tenemos la suficiente fuerza para romper la política del bipartidismo" @MailloAntonio en el acto de \#Málaga Con \#ElPoderdelaGente". 20-052014. Tuit.

No obstante, creemos que en el caso de UPyD se optó por utilizar las etiquetas \#MaitePagaza y \#SosaWagner para reproducir las declaraciones de ambos candidatos porque ninguno de ellos contaba con perfil en esta red social:

UPyD (@UPyD). “"Lo que democratas no hagamos para acoger e integrar a inmigrantes, lo harán xenófobos para expulsarlos" \#SosaWagner \#DebateEuropeasEnLaSER". 16-052014. Tuit.

Muchos políticos recurrieron también a etiquetas formadas por sintagmas nominales, como \#puroteatro o \#LasmentirasdeValenciano, para valorar las aserciones formuladas por los candidatos del PP y del PSOE en el transcurso del cara a cara entre ambos:

Willy Meyer (@willymeyerIU). "El no-debate fue una riña x las herencias, x el pasado A @iunida nos interesa el futuro \#elpoderdelagente \#puroteatro pic.twitter.com/ kDRTkKaK6M”. 17-05-2014. Tuit.

Partido (@PPopular). "Rajoy no ha concedido indultos por violencia de género frente a los 16 que dio Zapatero en su segundo mandato \#LasmentirasdeValenciano". 18-05-2014. Tuit.

Hashtags como estos cumplen una función valorativa, generalmente de contenido semántico negativo, algo que puede apreciarse también en las siguientes etiquetas, constituidas a partir de los sustantivos trampa o mordaza:

Teodoro García Egea (@TeoGarciaEgea).“@grumelude@SergiCastanye tu puedes decidir lo que quieras con lo que es tuyo, pero no con lo que es de todos los españoles. \#Trampa". 11-05-2014. Tuit.

\footnotetext{
${ }^{14}$ Sobre los distintos elementos que conforman el marco reproductor de los enunciados de discurso referido cfr. por ejemplo Girón $(1985,1988)$.
} 
Elena Valenciano (@ElenaValenciano). "Hoy se debate la moción de censura del PSOE de Extremadura al gobierno de Monago pero la televisión pública NO dará la sesión. \#PPMordaza". 14-05-2014. Tuit.

A juzgar por mensajes como el anterior, o como el que presentamos a continuación, donde se establece un juego de palabras con el hashtag \#Wertgüenza, la composición parece ser el mecanismo de creación léxica más frecuente en las etiquetas con función valorativa utilizadas por la candidata socialista:

Elena Valenciano (@ElenaValenciano). “¿Cuánto dinero dice Wert que van a ahorrar suprimiendi las becas de ayuda a niñ@s con autismo? Recortes contra moral! \#Wertgüenza". 12-05-2014. Tuit.

Véase también cómo para desacreditar la gestión del gobierno, Toni Cantó recurre a un acto de habla expresivo como es el agradecimiento, al que puede conferírsele una interpretación irónica, en virtud del cotexto previo:

Toni Cantó (@ToniCanto1). “40\% menos de huerta en Murcia en sólo 8 años \#GraciasPP pic.twitter.com/s01hyQ8T4K”. 21-05-2014. Tuit.

A veces, los políticos se sirven también de las etiquetas para formular enunciados como el siguiente, que se presenta como un acto de habla interrogativo:

Pablo Iglesias (@Pablo_Iglesias). “.@ElenaValenciano demuestra que no sois lo mismo que el PP. Sed coherentes y publicad el presupuesto de campaña ;) ¿\#QueTeneisQue Esconder?". 13-05-2014. Tuit.

En los mensajes que presentamos a continuación el enunciado que conforma el hashtag ha abandonado los signos de interrogación (de hecho, se ha prescindido de ellos al constituir la etiqueta), pero sigue ostentando fuerza exhortativa (cfr. Haverkate 2006):

Pablo Iglesias (@Pablo_Iglesias). “Cuéntame cuánto os gastáis @Canete2014_https:// www.youtube.com/watch?v=KgFE91GIcQE\&feature=youtu.be $\ldots \quad$ \#QueTeneisQue Esconder". 13-05-2014. Tuit.

Pablo Iglesias (@Pablo_Iglesias). “Porque tenemos que pedirle a @Canete2014_y @ElenaValenciano que digan cuanto se gastan firma \#QueTeneisQueEsconder https:// www.change.org/es/peticiones/miguel-arias-ca\%C3\%B1ete-y-elena-valenciano [...]". 1305-2014. Tuit.

Y adviértase cómo en este otro tuit el líder de Podemos apela a sus seguidores en lo que puede inferirse como una petición para que incluyan en sus mensajes el hashtag (HT) \#QueTeneisQueEsconder: 
Pablo Iglesias (@Pablo_Iglesias). “¿Me ayudáis a pedirle a @AriasCanete_ y @ElenaValenciano que publiquen los presupuestos de sus campañas con el HT \#QueTeneisQueEsconder?”. 13-05-2014. Tuit.

De esta forma entre todos lograrían que se convirtiera en trending topic, otorgando así mayor difusión a lo que implícitamente debe entenderse como un reproche a Arias Cañete y a Valenciano, por la opacidad de los presupuestos de campaña de sus respectivos partidos. Similar petición, aunque formulada de manera más explícita, encontramos en este otro mensaje enviado también por el líder de Podemos tres días después, aunque en esta ocasión la propia etiqueta adopta la forma exhortativa mediante el uso del imperativo del verbo difundir:

Pablo Iglesias (@Pablo_Iglesias). ¿Nos ayudas a convertir en TT \#DifundePodemos? Queremos hacer que Podemos sea visible en las calles. Recogemos propuestas y hacemos otras". 16-05-2014. Tuit.

Tampoco extraña que muchas de estas etiquetas constituyan actos de habla directivos con los que se incita a la participación mediante el uso del imperativo del verbo votar, que aparece fusionado con las siglas del partido para el que se solicita el apoyo:

Izquierda Unida (@iunida). “Somos la gente y tenemos el poder. @palomalopezB \#elpoderdelagente \#votaIU \#SomosIU". 23-05-2014. Tuit.

En el ejemplo precedente la etiqueta \#votaIU se yuxtapone a aquellas en las que se presentan los eslóganes del partido al final de un tuit publicado el último día de la campaña electoral, es decir, horas antes de que comience la jornada de reflexión. Resulta por tanto lógico que los partidos políticos aprovechen su última oportunidad para recabar apoyos en las redes sociales, finalizando la mayor parte de sus mensajes con el recuerdo de sus consignas e instando a la participación. De esa misma fecha data el siguiente tuit, aunque en este caso la etiqueta se integra en la apódosis de una oración condicional, en medio de una serie enumerativa conformada por tres verbos en modo imperativo:

Teodoro García Egea (@TeoGarciaEgea). "Si tienes selectividad pronto y puedes votar, busca un rato el domingo al medio día, \#VotaPP y sigue estudiando... SUERTE!!!”. 2305-2014. Tuit.

Mediante la segunda persona del singular el diputado popular apela directamente al alumno que está preparándose para el examen de acceso a la universidad (que tendrá lugar unas semanas después), tratando de persuadirle para que abandone brevemente su 
estudio en las horas centrales del día, con el fin de acudir a votar. Además, lo anima a perseverar en su tarea y le desea suerte, buscando de esa manera la captatio benevolentiae de un grupo de potenciales electores que participan por primera vez en unos comicios. No en vano, como demuestran las estadísticas ${ }^{15}$, son los jóvenes los que representan el mayor porcentaje de usuarios de las redes sociales. Sin embargo, lo habitual en este tipo de textos que incitan a la participación es que la etiqueta no se integre en el cuerpo del mensaje, sino que se presente a modo de eslogan final, recordando por ejemplo el nombre del candidato al adoptar el hashtag el esquema sintáctico <vota + nombre propio $>$ :

Partido Popular (@PPopular). “@@anete2014_: "El 25M los españoles tienen que dar una lección al socialismo" \#VotaCañete \#VotaPP http://www.pp.es/actualidad-noticia/ canete-dia-25-espanoles-tienen-que-dar-una-leccion-al-socialismo ...". 23-05-2014. Tuit.

Véase además cómo en este tuit las dos etiquetas poseen la misma orientación argumentativa que el enunciado de discurso referido del candidato número uno del PP a las elecciones europeas. De hecho, desde la cuenta oficial de dicho partido parece quererse sustentar con el contenido semántico de ambos hashtags la tesis precedente, formulada por Arias Cañete en el mitin de cierre de campaña mediante una perífrasis modal de infinitivo, en la que instaba a otorgar un "voto de castigo" al principal partido de la oposición. Por su parte, desde el perfil del PSOE, en lugar de hacer uso de la etiqueta \#votaValenciano ${ }^{16}$, se opta por reproducir lo que parecen ser las declaraciones de la candidata (si bien en este caso se ha omitido la marca de transposición discursiva que constituyen las comillas), haciéndolas preceder por el dispositivo @@nombredelusuario:

PSOE (@PSOE).“.@ElenaValenciano que ganen quienes lo están perdiendo todo \#TúMuevesEuropa Esta es nuestra propuesta para Europa \#VotaPSOE”. 19-05-2014. Tuit.

Es esta una convención del canal que garantiza que el mensaje llegue al usuario en cuestión, y que aparezca tanto en las cronologías de los seguidores del perfil oficial del partido como en la de los seguidores de Elena Valenciano. De esta forma se incrementa

\footnotetext{
${ }^{15}$ Cfr. por ejemplo el "V estudio anual de redes sociales" elaborado por Interactive Advertising Bureau. Disponible en: <http://www.iabspain.net/redes-sociales/>. Última consulta: 17-08-2014.

${ }^{16}$ Curiosamente, la herramienta de búsqueda de Twitter solo nos permite localizar un tuit en el que se hace uso de esta etiqueta: JJSS Plasencia (@JJSSdePlasencia). "Vamos compañeros, 3 días para las elecciones, \#VotaValenciano pic.twitter.com/JrVnUsIY2T”. 22-05-2014. Tuit.
} 
el número potencial de usuarios que recibirán el mensaje, de ahí que se opte también por recordar el principal eslogan con el que los socialistas concurren a las elecciones (esta es nuestra propuesta), que adopta en este caso el formato de hashtag, al presentar sus lexías fusionadas: \#TúMuevesEuropa. Dicha etiqueta ostenta también una vinculación argumentativa con el enunciado de discurso referido que le antecede, pues el uso del pronombre personal de segunda persona del singular parece servir para concretar el referente de la subordinada de relativo quienes lo están perdiendo todo. Por último, la etiqueta \#VotaPSOE multiplica también el alcance del tuit, haciendo que pueda ser recuperable por todos aquellos que accedan a dicho hiperenlace, susceptible de convertirse también en trending topic. Intención similar parece perseguir la diputada de UPyD Irene Lozano con el siguiente tuit:

Irene Lozano (@lozanoirene). “Os imagináis qué soledad si no pusiera 'Unión Europea'? Pues todo lo que no avanza retrocede. \#VotaEuropa @UPyD pic.twitter.com/ 7RUT288m0S". 15-05-2014. Tuit.

Dicho texto constituye un ejemplo de la relación sintagmática contextual (Menna 2012) a la que hacíamos alusión en el marco teórico. En primer lugar por el uso de la herramienta multimodal que constituye el hiperenlace que se presenta al final del mensaje. Este permite acceder a la fotografía de la cubierta de un pasaporte español, en la que pueden leerse claramente las palabras: Unión Europea España. Estas palabras constituyen el referente del verbo poner que aparece en el enunciado interrogativo inicial, cuya orientación argumentativa no puede comprenderse de manera cabal sin contemplar la imagen:

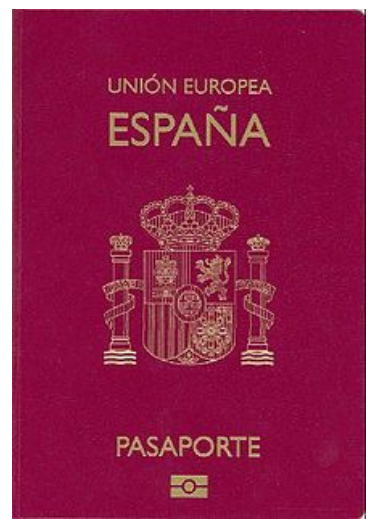

Por otra parte, llama la atención el hecho de que la parlamentaria se sirva inicialmente de la segunda persona del plural para apelar directamente a los lectores de su mensaje, y que opte después por la segunda persona del singular en la etiqueta \#VotaEuropa. Y es 
que la exposición de sus argumentos no cobra sentido pleno hasta la inclusión de dicho hashtag, que permite inscribir su texto en el marco cognitivo -en el sentido de Lakoff (2007) - de aquellos que ya desde el 9 de abril venían defendiendo la necesidad de acudir a votar en los comicios europeos, para evitar que unos elevados índices de abstención pudieran ser interpretados como oposición a la existencia de la Unión Europea. En segundo lugar, Lozano consigue hacer que sus razonamientos no solo se difundan entre sus seguidores, sino también entre aquellos que leen u opinan sobre ese mismo tema, inscribiendo su tuit en este ambiente de afiliación ya descrito por Zappavigna (2011). Esta, y no la petición del voto para su partido (si bien en yuxtaposición a dicha etiqueta incluye la referencia al perfil @UPyD), parece ser la finalidad principal de su mensaje, con el que se presenta a sí misma como partidaria de la integración europea, en oposición de aquellos que propugnan lo contrario. Y el mismo recurso argumentativo emplea en el siguiente tuit, cuyo texto posee también una alta dependencia contextual:

Irene Lozano (@lozanoirene). “Todos los políticos no son iguales... By Forges \#NoLesVotes PERO \#VotaAOtros. pic.twitter.com/Wov6Wy271G”. 18-05-2014. Tuit.

Así, la inclusión del adverbio de negación en el enunciado valorativo inicial permite interpretarlo como una contraargumentación directa del discurso referido que se presenta en la viñeta de Forges (Todos los políticos son iguales), a la que puede accederse mediante el hiperenlace situado al final del mensaje:



No obstante, los referentes de las etiquetas \#NoLesVotes y \#VotaAOtros resultan opacos para aquellos internautas que no estén familiarizados con el contexto electoral español. Por tanto, más que solicitar de manera explícita el voto para el propio partido, lo que pretende en este caso la diputada de UPyD es posicionarse entre aquellos que condenan el 
bipartidismo, abogando por la validez de otras opciones de gobierno. Esa misma tesis es defendida en el siguiente tuit por Toni Cantó, otro de los representantes de dicha formación política:

Toni Cantó (@ToniCanto1). “Felipe González no descarta Gobierno del PP apoyado por el PSOE, o al revés http://www.elconfidencial.com/espana/2014-05-12/felipe-gonzalez-nodescarta-un-gobierno-del-pp-apoyado-por-el-psoe-o-al-reves_128956/ ... Tanto monta/monta tanto \#StopBipartidismo". 12-05-2014. Tuit.

El mensaje comienza reproduciendo el titular de una noticia publicada en un confidencial, que se hace eco de unas declaraciones del ex presidente del Ejecutivo español Felipe González, en las que reconoce la posibilidad de que se forme un gobierno de coalición entre los dos partidos mayoritarios. Tales palabras, pronunciadas por una de las personas que ostenta mayor influencia en el partido socialista, parecen considerarse argumentos de autoridad que llevan al político de UPyD a concluir que las actuaciones políticas promovidas por PP y PSOE son muy similares. De ahí su uso del primer miembro discursivo de la conocida paremia que suele emplearse para manifestar la igualdad entre dos referentes: "Tanto monta monta tanto, Isabel como Fernando". Por último, el tuit concluye con la etiqueta \#StopBipartidismo, que incita a la acción de manera explícita mediante una estructura morfológica apelativa, y que en cierto modo podríamos considerar una muestra de intertextualidad, pues remite a uno de los hashtags más populares entre los usuarios de esta red social, símbolo de las actuaciones llevadas a cabo por la Plataforma de Afectados por la Hipoteca (PAH): \#StopDesahucios.

También desde el perfil oficial de UPyD se incide en la tesis de la semejanza entre los dos grandes partidos, mediante la etiqueta de estructura oracional \#PPSOEsonlomismo. Esta se presenta a modo de conclusión de la argumentación expuesta en los enunciados precedentes:

UPyD (@UPyD). “Sres Cañete y Valenciano ¿y de \#corrupción ni hablamos? pacto de hierro hasta en el \#CaraACaraTVE http://ow.ly/3ktFgJ \#PPSOEsonlomismo". 15-05-2014. Tuit.

En ellos se increpa tanto al candidato popular como a la candidata socialista para dirigirles una pregunta retórica en la que se ha recurrido al símbolo de la etiqueta para otorgar mayor fuerza argumentativa al sustantivo de carga semántica negativa corrupción. Adviértase que tanto esta etiqueta como la de \#CaraACaraTVE desempeñan funciones sintagmáticas, la de complemento de régimen preposicional y la de complemento circunstancial, 
respectivamente, y se encuentran perfectamente integradas en las estructura oracional del tuit. Y lo mismo sucede en este otro mensaje, en el que se aprovecha la estructura del eslogan de Podemos para conformar una perífrasis modal de infinitivo:

Podemos (@ahorapodemos). “\#VotandoPodemos evitar que nuestros jóvenes carezcan de un futuro digno en nuestro país. https://www.youtube.com/watch?v=-CC3K68f9-g ...". 22-052014. Tuit.

Si consideramos la posición en la que se colocan este tipo de etiquetas similares a un eslogan de partido, observamos que a veces se integran al comienzo del mensaje, lo que confiere mayor realce a su contenido semántico:

Pablo Iglesias (@Pablo_Iglesias).“\#YoVotoPodemos Acaban de publicar esta entrevista que me han hecho en El País http://politica.elpais.com/politica/2014/05/23/actualidad/ 1400863156_284091.html ...”. 23-05-2014. Tuit.

Podemos (@ahorapodemos). “\#YoVotoPodemos Mira este vídeo genial que nos han mandado con imágenes del SextoSentido :) http://www.youtube.com/watch?v=Na1X8 EvcXOU\&feature=youtu.be ...". 23-05-2014. Tuit .

Además, en ocasiones, el hashtag puede aparecer también situado en el margen izquierdo de la oración para ejercer una función tematizadora. Véase cómo en el siguiente ejemplo la etiqueta en la que se especifica el nombre propio de uno de los candidatos precisa el contenido referencial del sintagma preposicional "del candidato de @UPyD”:

UPyD (@UPyD). “\#SosaWagner la tributación transparente del eurodiputado de @UPyD http://ow.ly/x2Jah pagando siempre todos sus impuestos en España”. 20-05-2014. Tuit.

No obstante, tal estrategia parece poco frecuente, pues solo se utiliza en 32 de las 1.970 etiquetas de nuestro corpus, es decir, en el 1,62\% de los casos (19 en los perfiles de representantes políticos y 13 en los de los partidos). Más habitual es que las etiquetas se encuentren integradas en la estructura oracional del mensaje, en el seno del cual realizan una determinada función sintáctica, generalmente, de complemento circunstancial cuando indican la ciudad o el evento (mitin, debate televisado) al que remite el contenido del tuit. Algo que hemos podido comprobar en 239 etiquetas publicadas en los perfiles de políticos y en 118 difundidas en los de los partidos, lo que suma un total de 357 hashtags, que representan el $18,12 \%$ del total. Pero, sin lugar, a dudas la práctica más usual consiste en situar la etiqueta al final del mensaje, y en estos casos esta reproduce el eslogan del partido político o adopta una estructura exhortativa con la que se solicita la difusión de una determinada idea o el voto de los ciudadanos. Dicha estructura es especialmente recurrente en las cuentas de las formaciones políticas (1.127), si bien también predomina en las de los líderes políticos (454), lo que suma un total de 1.581 etiquetas, que tienen una representatividad del 80,25\%. 


\section{Conclusiones}

El análisis llevado a cabo nos permite dar respuesta a las cuestiones planteadas al inicio de este estudio. En primer lugar, es posible afirmar que los hashtags de nuestro corpus establecen relaciones sintagmáticas internas (Menna 2012) con los elementos lingüísticos y semióticos que aparecen en los tuits, situándose tanto al inicio del mensaje como al final del mismo. En los casos en los que aparecen al final, más numerosos, suelen presentarse como sintagmas integrados en una oración con funciones sintácti\#cas de complemento circunstancial, para informar sobre el lugar de un evento o indicar el contexto en el que se deben situar unas declaraciones. Así, en el plano del discurso, estos tienen fundamentalmente funciones organizadoras y contextualizadoras. Por ejemplo, en las etiquetas relativas al debate predomina esta última función, que contribuye además a crear un ambiente de afiliación (Zappavigna 2011) que permite al usuario saber qué se dice en Twitter sobre el cara a cara. Por otra parte, las etiquetas presentan relaciones sintagmáticas externas cuando reenvían, por ejemplo, a una imagen o noticia que complementa el contenido del mensaje, y contextuales, en la medida en que, por ejemplo, a través de la ironía generan nuevos significados que remiten al contexto situacional de la campaña y que apelan a los conocimientos compartidos por el enunciador y el enunciatario. En segundo lugar, los hashtags analizados tienen una clara función persuasiva en el discurso político electoral, lo que confirma los resultados obtenidos por Mancera y Pano (2013) relativos a la campaña electoral española de 2011, y los del estudio de Mancera y Helfrich (2014) sobre la retórica de la persuasión de las etiquetas. En estos casos se sitúan al final del tuit como sintagmas con connotaciones tanto apelativas (por ejemplo, en el caso de los hashtags del tipo <vota + nombre propio $>$ ) como negativas, o en estructuras oracionales con predicado verbal o nominal. En general, comparables a los eslóganes o las consignas, la mayor parte de las etiquetas analizadas sirven a los políticos para criticar al adversario o a su partido y, cuando vehiculan actos exhortativos, les permiten llamar a los ciudadanos a la acción, a denunciar un estado de cosas, o a votar. 
Bibliografía

Chew, C. y G. Eysenbach (2010): Pandemics in the age of Twitter: content analysis of tweets during the 2009 H1N1 outbreak. PLoS ONE 5 (11). < http://journals. plos.org/plosone/article?id=10.1371/journal.pone.0014118 > . Última consulta: 23-072014.

Cunha, E. y otros (2011): Analyzing the dynamic evolution of hashtags on Twitter: a language-based approach. En: Proceedings of the Workshop on Language and Social Media, Portland, Oregon, 58-65. < http://www.aclweb.org/anthology/W11-0708 >. Última consulta: 24-07-2014.

Dann, S. (2010): Twitter content classification. First Monday 15 (12). < http://firstmon day.org/ojs/index.php/fm/article/view/2745/2681 >. Última consulta: 25-07-2014.

Davis, B. (2013): Hashtag politics: the polyphonic revolution of \#Twitter. Pepperdine Journal of Communication Research 1 (4), 15-22. < http://digitalcommons.pepper dine.edu/pjcr/vol1/iss1/4 > Última consulta: 02-03-2015.

Girón Alconchel, J. L. (1985): La 'escritura del habla' y el discurso indirecto libre en español. Archivo de Filología Aragonesa (AFA) 36-37, 172-204.

Girón Alconchel, J. L. (1988): La reproducción del discurso en la lengua hablada. En: Asociación Española de Semiótica (ed.), II Simposio Internacional de Semiótica. Lo cotidiano y lo teatral, Oviedo: Servicio de Publicaciones de la Universidad de Oviedo, 203-215.

Haverkate, H. (2006): Aspectos pragmalingüísticos de la interrogación en español con atención especial a las secuencias de preguntas. Cultura, Lenguaje y Representación $3,27-40$.

Lakoff, G. (2007): No pienses en un elefante: lenguaje y debate político. Madrid: Complutense.

López Eire, A. y J. de Santiago Guervós (2000): Retórica y comunicación politica, Madrid: Cátedra.

Mancera Rueda, A. y U. Helfrich (2014): La crisis en 140 caracteres: el discurso propa- 
gandístico en la red social Twitter. Cultura, Lenguaje y Representación 12, 59-86.

Mancera Rueda, A. y A. Pano Alamán (2013): El discurso político en Twitter. Barcelona: Anthropos.

Menna, L. (2012): Nuevas formas de significación en red: el uso de las \#etiquetas en el movimiento 15M. Estudios de Lingüística del Español 34. < http://elies.rediris.es/ elies34/Tesina_L-Menna.pdf >. Última consulta: 24-07-2014.

Page, R. (2012): The linguistics of self-branding and micro-celebrity in Twitter: the role of hashtags. Discourse \& Communication 6, 181-201.

Pano Alamán, A. y A. Mancera Rueda (2014): La 'conversación' en Twitter: las unidades discursivas y el uso de marcadores interactivos en los intercambios con parlamentarios españoles en esta red social. Estudios de Lingüística del Español 35, 243-278. < http://infoling.org/elies/35/elies35.1-10.pdf >. Última consulta: 28-072014.

Parmelee, J. H. y S. L. Bichard (2012): Politics and the Twitter Revolution. How Tweets influence the relationship between political leaders and the public. Londres, Lexington Books.

Pérez Fumero, E. (2013): La enfermedad de Hugo Chávez en la agenda de medios y públicos. Análisis del 'hashtag' \#ElMundoEstaconChavez desde la teoría del 'framing'. Index.Comunicación 3, 63-85.

Reyes, A. y otros (2012): From humor recognition to irony detection: the figurative language of social media. Data \& Knowledge Engineering 74, 1-12.

Romero, D. y otros (2011): Differences in the mechanics of information diffusion across topics: idioms, political hashtags, and complex contagion in Twitter. International WWW Conference. < http://www.cs.cornell.edu/home/kleinber/www11-hashtags. pdf > . Última consulta: 22-07-2014.

Shaffer, E. y otros (2013): Tweeting the Government: Preliminary findings from a genre analysis of Canadian federal government tweets. GRAND, May 14-15, Toronto. $<$ http://works.bepress.com/cgi/viewcontent.cgi?article=1022\&context=elizabeth_sha ffer $>$. Última consulta: 27-07-2014. 
Tsur, O. y A. Rappoport (2012): What's in a hashtag? Content-based prediction of the spread of ideas in microblogging communities. WSDM'12, February 8-12, Seattle, Washington. < http://eprints.pascal-network.org/archive/00009315/01/wsdm308tsur.pdf >. Última consulta. 23-07-2014.

Zappavigna, M. (2011): Ambient affiliation: a linguistic perspective on Twitter. New Media Society 13 (5), 788-806.

Zappavigna, M. (2012): Discourse of Twitter and Social Media. Londres: Continuum.

Recibido: 22 de agosto de 2014 Aceptado: 21 de noviembre de 2014 Publicado: 30 de noviembre de 2015 